\title{
A RESTRICTION THEOREM FOR THE FOURIER TRANSFORM
}

\author{
BY PETER A. TOMAS
}

Communicated by Harry Kesten, October 24, 1974

Let $f$ be a Schwartz function on $\mathbf{R}^{n}$, and let $\hat{f}(\theta)$ denote the restriction of the Fourier transform of $f$ to the unit sphere $S^{n-1}$ in $\mathbf{R}^{n}$. We prove

THEOREM. If $f$ is in $L^{p}\left(\mathbf{R}^{n}\right)$ for some $p$ with $1 \leqslant p<2(n+1) /(n+3)$, then

$$
\int_{S^{n-1}}|\hat{f}(\theta)|^{2} d \theta \leqslant c_{p}\|f\|_{p}^{2}
$$

ProOF.

$$
\int|\hat{f}(\theta)|^{2} d \theta=\int f * \widetilde{f}(x) \widehat{d \theta}(x) d x=\int f(x) \widehat{d \theta} * f(x) d x \leqslant\|f\|_{p}\|\widehat{d \theta} * f\|_{p^{\prime}}
$$

for conjugate indices $p$ and $p^{\prime}$. Thus it suffices to prove that the operator given by convolution with $\widehat{d \theta}$ is bounded from $L^{p}$ to $L^{p^{\prime}}$ for $p$ in the appropriate range. Let $K(x)$ be a radial Schwartz function with $K(x)=1$ for $|x| \leqslant 100$, and let $T_{k}(x)=\left[K\left(x / 2^{k}\right)-K\left(x / 2^{k-1}\right)\right] \widehat{d \theta}(x)$. It suffices to show there exists $\epsilon=\epsilon(p)>0$ such that $\left\|T_{k} * f\right\|_{p^{\prime}} \leqslant C 2^{-\epsilon k}\|f\|_{p}$. This follows from interpolating the estimates $\left\|T_{k} * f\right\|_{\infty} \leqslant C 2^{-(n-1) k / 2}\|f\|_{1}$ and $\left\|T_{k} * f\right\|_{2} \leqslant$ $2^{k}\|f\|_{2}$.

Professor E. M. Stein has extended the range of this result to include $p=2(n+1) /(n+3)$. His proof uses complex interpolation of the operators given by convolution with the functions $B_{\sigma}(x)=J_{\sigma}(2 \pi|x|) /|x|^{\sigma}$. Then $\widehat{d \theta}(x)=B_{(n-2) / 2}(x)$.

A great deal was previously known about such restriction theorems. E. M. Stein originally established the theorem for $1 \leqslant p<4 n /(3 n+1)$. For $n=2$, this was extended by Fefferman and Stein [2] to the range $1 \leqslant p<$ 6/5. P. Sjolin (see [1]) proved the theorem for $n=3$ and $1 \leqslant p \leqslant 4 / 3$. Finally, A. Zygmund [3] determined for two dimensions all $p$ and $q$ such that the Fourier transform of an $L^{p}$ function restricts to $L^{q}\left(S^{1}\right)$. Since a $42 \mathrm{~A} 18$.

$A M S$ (MOS) subject classifications (1970). Primary 42A68, 42A92; Secondary 
very simple homogeneity argument shows that the theorem fails for $p>2(n+1) /(n+3)$, the present result, together with the result of Stein, represent the optimal estimate of this sort.

I am deeply grateful to Professor A. W. Knapp whose many comments and criticisms were invaluable in proving this result.

\section{REFERENCES}

1. L. Carleson and P. Sjolin, Oscillatory integrals and a multiplier problem for the disc, Studia Math. 44 (1972), 287-299.

2. C. Fefferman, Inequalities for strongly singular convolution operators, Acta Math. 124 (1970), 9-36. MR 41 \#2468.

3. A. Zygmund, On Fourier coefficients and transforms of functions of two variables, Studia Math. 50 (1974), 189-201.

DEPARTMENT OF MATHEMATICS, PRINCETON UNIVERSITY, PRINCETON, NEW JERSEY 08540 